\title{
Scene Illumination as an Indicator of Image Manipulation
}

\author{
Christian Riess` and Elli Angelopoulou \\ Pattern Recognition Lab \\ University of Erlangen-Nuremberg \\ \{riess, angelopoulou\}@i5.cs.fau.de \\ http://www5.informatik.uni-erlangen.de
}

\begin{abstract}
The goal of blind image forensics is to distinguish original and manipulated images. We propose illumination color as a new indicator for the assessment of image authenticity. Many images exhibit a combination of multiple illuminants (flash photography, mixture of indoor and outdoor lighting, etc.). In the proposed method, the user selects illuminated areas for further investigation. The illuminant colors are locally estimated, effectively decomposing the scene in a map of differently illuminated regions. Inconsistencies in such a map suggest possible image tampering. Our method is physics-based, which implies that the outcome of the estimation can be further constrained if additional knowledge on the scene is available. Experiments show that these illumination maps provide a useful and very general forensics tool for the analysis of color images.
\end{abstract}

Keywords: Blind image forensics, scene analysis, physics-based illuminant color estimation

\section{Introduction}

The goal of image forensics is to assess image authenticity. This can be achieved by actively embedding a security scheme in the image, like a digital watermark. However, current hardware does not typically provide such signatures. Therefore, blind image forensics aims at assessing image authenticity and origin without having an embedded security scheme available. In the past few years, different branches of blind image forensics have evolved.

For a more complete overview on the methods in blind image forensics, see e.g. [37, 33].Some of the existing approaches are classification based [6, 22]. Unfortunately, the outcome of these algorithms is often hard to interpret for nontechnical surveyors, e.g. in court. Other approaches search for artifacts of a specific tampering operation, like the algorithms for copy-move forgery detection (see e.g. [20, 2, 3, 32, 31]), resampling detection [35], or methods for the analysis of double JPEG compression (see e.g. [29, 15, 7]). In general, these methods are suitable for an automated analysis of an image. Unfortunately, researchers are also

\footnotetext{
* Corresponding author
} 
working on methods to hide image manipulation artifacts, thus effectively counteracting the aforementioned methods [23]. A third family of algorithms aims at verifying expected image-sensing artifacts. One prominent example of these methods is the recovery of the characteristic noise pattern of camera sensors [30]. Other methods estimate the camera response function [17], demosaicing [12], or lens properties [21].

Lastly, the examination of scene properties is another approach in image forensics. Unlike the aforementioned methods, it often involves (to a limited extend) user interaction, especially if human knowledge about the scene content is required. In this respect, methods for the assessment of scene properties often serve as a computational tool for a human surveyor. The advantage of such techniques is that it is frequently not straightforward to hide traces of tampering from these methods. Disguising scene inconsistencies is typically a tedious manual process, that may additionally require high algorithmic knowledge. Thus, scene consistency assessment can provide powerful tools for forensic analysis. To our knowledge, only a small amount of work has been done in this direction. For instance, Johnson and Farid demonstrated the recovery of the illumination direction of objects [19] and compared the estimated position of light sources in the scene [18]. Yu et al. examined specularity distributions for recapturing detection [40]. Lalonde and Efros used color distributions in pictures in order to detect spliced images [25].

We propose a new method for the assessment of illumination-color consistency over the scene by extracting local illumination estimates. To our knowledge, no similar approach has been proposed in image forensics. Our method is based on an extension of an illumination estimation method that is grounded on the physical principles of image formation. In contrast, most state-of-the-art methods for illuminant color estimation are machine-learning based. However, it is our belief that deviations from the expected result can be easier explained using a physics foundation than by machine-learning results, as detailed in Sec. 4.2. We believe this is a highly desirable property in forensics applications. Depending on the number of light sources of the scene, we show that these local estimates can provide further insights on the scene construction. For instance, if a photographer took an image at night using flashlight (which is typically a relatively bluish light source), we can obtain a rough relative depth estimate from the decay of the blue channel in the illuminant estimates. Inconsistencies in the illumination distribution can be used to distinguish original and spliced images.

The contributions of this paper are:

1. The development of a physics-based method for the recovery of the illuminant color for different objects in the scene.

2. The introduction of an illumination map based on a distance measure on the estimated results.

3. The demonstration of the feasibility of employing this illuminant map in forensic analysis. 


\section{Overview of the Method}

We present a system for the assessment of the illuminant color consistency over the image. The method involves the following steps.

1. The image is segmented in regions of approximately the same object color. These segments are called superpixels. A superpixel is required to a) be directly illuminated by the light sources under examination and b) roughly adhere to the physical model presented in Sect. 3.

2. A user selects such superpixels whose incident illuminant he wants to further investigate. Every group of superpixels represents one illuminant color under investigation.

3. Estimation of the illuminant color is performed twice. First, the estimation is done on every superpixel separately. Second, the estimation is done on the user-selected superpixel groups for greater robustness.

4. The user-selected groups form the reference illuminants. A distance measure from these illuminants to every superpixel estimate is computed. We visualize these per-superpixel distances in what we call a distance map to support the analysis of the illumination color consistency.

In special cases, this method can be fully automated. On the other hand, since the estimation of the illuminant color is an underconstrained problem, there will always exist scenes that can not be correctly processed. We believe that a limited degree of human interaction is a valid tradeoff between the accuracy of the method and its usability.

\section{Estimation of the Illuminant Color}

There is a large body of work on the estimation of illuminant color. Most of these methods process the entire scene globally for the recovery of a single dominant illumination color. To overcome the fact that illuminant color estimation is an underconstrained problem, many (especially physics-based) techniques make restrictive assumptions that limit their applicability (e.g. [26,13, 24]). As a result, machine learning methods have been more successful in processing arbitrary images, e.g. $[4,9,10,14,28]$. However, since illuminant estimation is an underconstrained problem, every method has its individual failure points. We chose a physics-based method, since the failures typically result from broken underlying assumptions. As such, they can (by human observers) more easily be predicted and explained, which we consider highly important for forensics applications.

\subsection{Inverse-Intensity Chromaticity Space}

We extend a physics-based approach that was originally proposed by Tan et al [39] so that:

1. it can be applied on a wider range of real-world images, and 
2. it can be applied locally, so that illuminants at selected regions can be independently estimated.

The foundation of [39] is the dichromatic reflectance model [38], which states that the amount of light reflected from a point, $\boldsymbol{x}$, of a dielectric, non-uniform material is a linear combination of diffuse reflection and specular reflection. Further assumptions are that the color of the specularities approximates the color of the illuminant, and that the camera response is linear.

When an image is taken with a trichromatic camera, the sensor response $I_{c}(\mathbf{x})$ for each color filter $c, c \in\{R, G, B\}$ is:

$$
I_{c}(\mathbf{x})=w_{d}(\mathbf{x}) B_{c}(\mathbf{x})+w_{s}(\mathbf{x}) G_{c}(\mathbf{x}),
$$

where $w_{d}(\mathbf{x})$ and $w_{s}(\mathbf{x})$ are geometric parameters of diffuse and specular reflection respectively. $B(\mathbf{x})$ and $G_{c}(\mathbf{x})$ are the sensor responses for diffuse and specular reflectance. Note that in [39], $G_{c}$ does not depend on $\mathbf{x}$ due to the assumption that the specular color is globally constant. In this paper, we estimate illumination locally and thus write $G_{c}(\mathbf{x})$. Let $\sigma_{c}$ the image chromaticity, i.e.

$$
\sigma_{c}(\mathbf{x})=\frac{I_{c}(\mathbf{x})}{\sum_{i} I_{i}(\mathbf{x})} \text { where } i \in\{R, G, B\} .
$$

For the remainder of the paper, we define $i \in\{R, G, B\}$ and use this index for summing over the color channels. In a similar manner, we can define the diffuse chromaticity $\Lambda_{c}(\mathbf{x})$ and the specular chromaticity $\Gamma_{c}(\mathbf{x})$ as

$$
\begin{aligned}
& \Lambda_{c}(\mathbf{x})=\frac{B_{c}(\mathbf{x})}{\sum_{i} B_{i}(\mathbf{x})}, \\
& \Gamma_{c}(\mathbf{x})=\frac{G_{c}(\mathbf{x})}{\sum_{i} G_{i}(\mathbf{x})} .
\end{aligned}
$$

Equation (1) can be rewritten as

$$
I_{c}(\mathbf{x})=m_{d}(\mathbf{x}) \Lambda_{c}(\mathbf{x})+m_{s}(\mathbf{x}) \Gamma_{c}(\mathbf{x}),
$$

where

$$
\begin{aligned}
& m_{d}(\mathbf{x})=w_{d}(\mathbf{x}) \sum_{i} B_{i}(\mathbf{x}), \\
& m_{s}(\mathbf{x})=w_{s}(\mathbf{x}) \sum_{i} G_{i}(\mathbf{x}) .
\end{aligned}
$$

Tan et al. [39] showed, that there exists a linear relationship between diffuse, specular and image chromaticities,

$$
\sigma_{c}(\mathbf{x})=p_{c}(\mathbf{x}) \frac{1}{\sum_{i} I_{i}(\mathbf{x})}+\Gamma_{c}(\mathbf{x})
$$

where

$$
p_{c}(\mathbf{x})=m_{d}(\mathbf{x})\left(\Lambda_{c}(\mathbf{x})-\Gamma_{c}(\mathbf{x})\right) .
$$


$p_{c}(\mathbf{x})$ is the slope of a line with intercept $\Gamma_{c}(\mathbf{x})$, i.e. the specular chromaticity, which is also the illuminant chromaticity. The domain of the line is determined by $1 / \sum_{i} I_{i}(\mathbf{x})$ and the range is given by $0 \leq \sigma_{c} \leq 1$. Domain and range together form the inverse-intensity chromaticity (IIC) space [39].

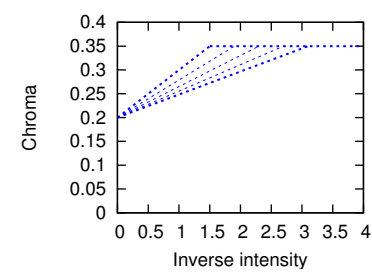

(a)

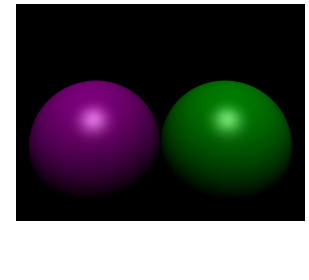

(b)

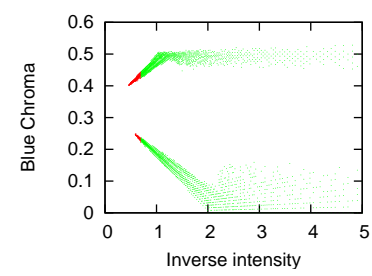

(c)

Fig. 1. Sample pixel distributions in IIC space (blue chromaticity). Left: ideal image, middle: synthetic image (violet and green bowls). Right: specular pixels converge towards to the blue portion of the illuminant color (recovered at at the y-axis intercept). Highly specular pixels are shown in red.

This space can be used to estimate the illumination color in an image. Every color band $c \in\{R, G, B\}$ is projected in a separate IIC diagram. One can then obtain estimates for each of these three color channels. All three channels together form the illuminant estimate. Fig. 1 shows a synthetic example for the blue channel. The $x$-axis corresponds to the inverse-intensity $1 / \sum_{i} I_{i}(\mathbf{x})$, and the $y$-axis to $\sigma_{c}$. In Fig. 1 the $y$-axis shows the blue chromaticities. An idealized distribution is shown in Fig. 1(a). On a uniformly colored surface, pixels with a mixture of specular and diffuse chromaticities form roughly a triangle that points towards the illuminant chromaticity (the $y$-axis intercept). Purely diffuse pixels form a straight line. Fig. 1(b) shows a rendered image of two balls of distinct colors, and Fig. 1(c) shows the distributions of the balls-image in the blue chromaticity IIC space. In this synthetic setup, the triangles can be clearly captured, as well as the diffuse horizontal lines. Any method for finding the intersection of the triangle with the $y$-axis gives then the illuminant chromaticity estimate in the respective channel. The final estimate of the illuminant color is obtained by considering all three color bands red, green and blue. In [39], this is done by first segmenting highly specular pixels in the image (marked red in Fig. 1(c)) and then performing a Hough transform on these specular pixels.

\subsection{Local Analysis of Pixel Distributions}

On real-world images, the automatic extraction of highly specular pixels is a very challenging task with unreliable performance (see e.g. [36]). Furthermore, the basic method [39] does not handle cases with multiple light sources. We 
analyzed and extended the exploitation of pixel distributions in IIC space, so that it can overcome these two weaknesses.

In order to avoid specularity segmentation, we chose to perform simple shape checks on the pixel distributions in IIC space in order to identify specular regions. Instead of examining the entire pixel distribution, we perform the analysis over small connected image regions of roughly uniform object color (albedo). Depending on the outcome of our shape analysis, we can either use this local region to obtain an illuminant estimate, or reject it if it does not seem to fulfill the underlying assumptions of the proposed model. Using local regions allows us to incorporate multiple sampling and voting in the estimation of local illuminants. Ultimately, this improved exploitation of the IIC space makes the method more robust to real-world analysis and also enables us to examine multiple illuminants. More specifically, the proposed algorithm works as follows.

1. For every dominant illuminant in the scene, select regions that a) follow the dichromatic reflectance model and b) are mostly lit by that light source.

2. Segment these regions in superpixels with roughly uniform chromaticity.

3. Further subdivide these superpixels in a rectangular grid. We call each such rectangular subregion a patch.

4. Transform every patch to inverse intensity space.

5. Apply tests on the shape of the patch's pixel distribution. If the distribution passes, obtain a local illuminant color estimate for this patch.

6. Obtain a color estimate for each dominant illuminant, based on a majority vote on local estimates of the user-selected regions.

For the superpixel segmentation, we used the publicly available code by Felzenszwalb and Huttenlocher [8] on the image chromaticities, though any segmentation method could be used. We choose the segmentation parameters $0.1 \leq \sigma \leq 0.3$ and $100 \leq k \leq 300$. Typically $\sigma=0.3$ and $k=300$ gave satisfying results, dividing the image in not too small regions of similar object color. The grid size is adaptive to the image size, typically between 16 and 32 pixels in the horizontal and vertical directions.

Pixel distributions that are assumed to exhibit a combination of specular and diffuse reflectance should have: a) a minimum $x$-axis elongation in IIC space, and b) a non-horizontal slope (as long as the object albedo is different from the illuminant color, which is typically the case). Thus, we use the following criteria to examine whether a patch satisfies these two properties and hence has an increased probability of providing a reasonable estimate.

- The superpixel segmentation is performed for increasing the probability of uniform underlying albedo.

- The elongation of a patch in IIC space is tested by computing the eccentricity of the distribution of the pixels in the patch,

$$
\operatorname{ecc}\left(P_{\mathrm{IIC}}\right)=\sqrt{1-\frac{\sqrt{\lambda_{1}}}{\sqrt{\lambda_{2}}}},
$$


where $\lambda_{1}$ and $\lambda_{2}$ are the largest and second largest eigenvalues, respectively. A value close to 1 is matches the model best, while lower values lead to more estimates. In our experiments, we chose as a limit 0.6.

- The slope of a patch in IIC space is approximated by the direction of the eigenvector $\lambda_{1}$, and should be slightly larger than 0 . In our experiments, we chose a minimum slope of 0.003 .

The vote for a patch is computed as the intercept of the eigenvector of $\lambda_{1}$ with the $y$-axis. Note that duplicate entries in the IIC diagram are discarded from these computations, as well as pixels that are very close to the limits of the camera sensor response.

\section{Illuminant Color for Image Forensics}

Once the illuminant color estimates for the user-annotated regions are computed, the whole image can be examined for illumination color inconsistencies, as described in Sect. 4.1. Since the estimation of the illuminant color is a severely underconstrained problem, we briefly discuss failure cases and possible workarounds in Sect. 4.2. Please note that, as will be shown in Sect. 5, our illumination estimation method performs comparably to other state-of-the-art single illuminant estimation methods.

\subsection{Detecting Inconsistencies in Illumination}

The same process (see Sect. 3.2) that was used in computing the illuminant color estimates at the user-specified regions is now extended to the entire image. The voting, however, is now performed for every superpixel. Thus, every superpixel contains an individual illuminant estimate. We store these illuminant estimates in a new image, where each superpixel is colored according to its estimated illuminant color $\Gamma_{I}(\mathbf{x})$. We call this new image illumination map, see Fig. 2. This map gives already quite meaningful results for the analysis.

For forensic analysis, we aim to quantify the relationship between the illuminant estimates. In a scene with truly one dominant illuminant, this can be done by comparing the angular errors of the individual illuminant estimates. However, most real-world scenes contain a mixture of illuminants. Their influence on the scene is closely connected to the positions of the objects relative to the positions of the light sources. Since the geometric composition of the scene is typically unknown, we resort to developing a tool for supporting the visual assessment of the scene, which we call distance map.

The distance map captures how well the illuminant estimation at each superpixel fits to the estimated dominant illuminants. For improved clarity, we assume two dominant illuminants $I_{1}$ and $I_{2}$ that were obtained from two user-selected regions. The methodology can however easily generalize to more illumination sources. We aim to create a grayscale-image that depicts the relative influence of both light sources. The distance map is created by assigning the value 0 (black) 
to the user-defined region corresponding to illuminant $I_{1}$. Similarly, the second user-defined region, which gave rise to dominant illuminant $I_{2}$, is assigned the value 1 (white). Then, for all the remaining pixels, the distance value $I_{d}(\mathbf{x})$ is computed as

$$
I_{d}(\mathbf{x})=\left(\Gamma_{I}(\mathbf{x})-I_{1}\right) \circ\left(I_{2}-I_{1}\right),
$$

where $\circ$ denotes scalar multiplication. The distance map is then a grayscale image with values in the range $[0,1]$. Such a map captures the relative influence of both light sources in this pixel.

The illumination map and the distance map are used together for the analysis of the image. In order to be consistent, a local illuminant estimate in an image must a) either exhibit a relative illuminant contribution that fits in the spatial layout of the scene or b) fail to fulfill the underlying physical model. In the latter case, it must be ignored for the analysis.
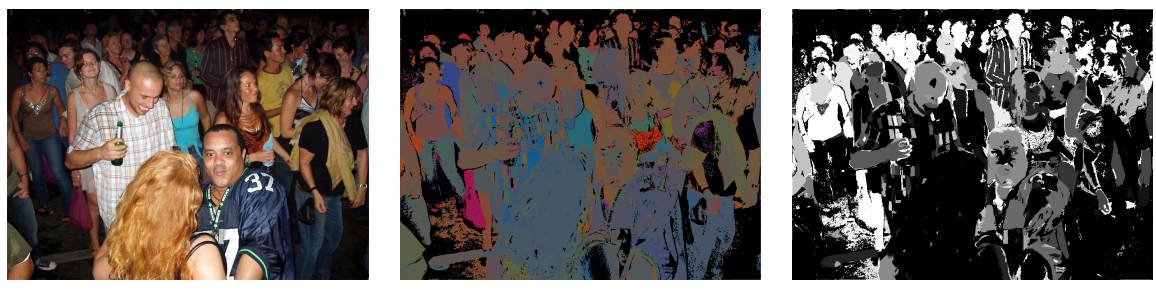

Fig. 2. Original Image, illumination map and distance map for the image under examination. Foreground persons are estimated with a bluish color, probably due to flashlight, while persons in the background are increasingly red illuminated. The distance map between foreground and background illumination spots captures this relationship as a black-to-white transition.

By adjusting the values of the criteria on the pixel distributions, it is possible to obtain fewer estimates that fit the physical model better (at the expense of larger regions with sparse or no estimates). Alternatively, less strict parameters lead to a more complete map, where also more outliers are expected. In general, we preferred in our experiments lenient settings. For the slope we set a lower bound of 0.003, and for the eccentricity 0.5. A stricter set of values, i.e. 0.01 for the slope and 0.95 for the eccentricity, typically results in fewer outliers.

\subsection{Caveats and Workarounds}

In some cases, the estimation of the illuminant color can not be successfully applied. Fortunately, for a physics-based method like the proposed one, the reasoning about failure cases is often easier than for machine-learning methods. While failures in the latter case often arise due to limitations of the training data or algorithm-dependent assumptions on the color distributions, physics-based 
methods mainly fail due to violations of the assumed reflectance model. This makes it possible to argue about possible problems and look for workarounds.

We present some cases where our method is problematic. First, the camera response is assumed to be linear. This is leveraged by the fact that we exploit only the relationship between illuminant estimates, and do not consider absolute estimates. Nevertheless, a gamma estimation method, e.g. [27], can be used to normalize the image. Some non-dielectric surfaces are especially difficult to handle, e.g. fluorescent materials (see Fig. 3) and metals. Other failure cases involve

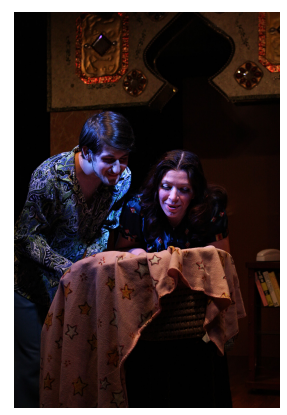

(a)

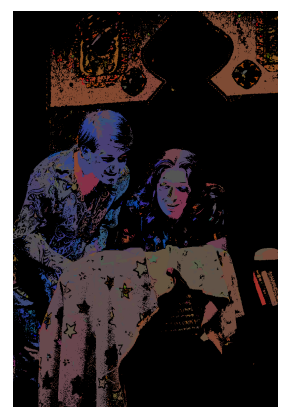

(b)

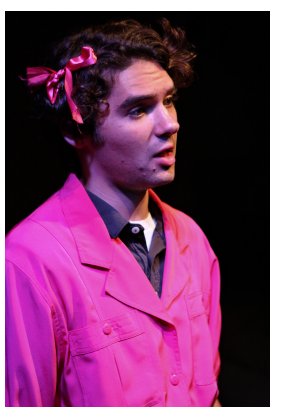

(c)

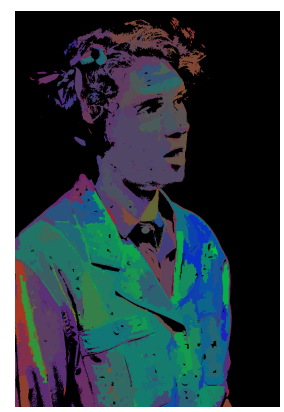

(d)

Fig. 3. Failure cases for the proposed illuminant color estimation method. Figures 3(a) and $3(\mathrm{c})$ are the original images, Figures $3(\mathrm{~b})$ and $3(\mathrm{~d})$ the respective illumination maps. In Fig. 3(b), the illuminant estimate in the shadowed area under the head of the left actor is biased towards the object color. In Fig. 3(d), the fluorescent suit of the actor overproportionally pushes the illuminant estimate towards extreme values.

areas that are mostly diffuse, or highly textured, or in shadow (see Fig. 3). Finally, the method is inherently limited by the assumption that the color of the specularity closely approximates the color of the illuminant.

We found, that by visual inspection it is often possible to distinguish failure cases from real inconsistencies. However, it is possible to follow specific rules to minimize the risk of misjudging the scene under observation. The most robust approach is to use only identical or very similar materials for the analysis, e.g. faces in a crowded scene. We reflect this by demanding the user to select regions that a) are of interest for the examination and b) roughly adhere to the model.

\section{Experiments}

Section 5.1 demonstrates the effectiveness of our core algorithm in accurately recovering the color of the illumination. In Sect. 5.2, we demonstrate its usefulness in forensics applications. The code is publicly available on our webpage ${ }^{1}$.

\footnotetext{
${ }^{1}$ http://www5.informatik.uni-erlangen.de/code
} 


\subsection{Evaluation on Benchmark Databases}

Until today, the color research community focused mostly on single-illuminant scenes. Therefore, to the best of our knowledge, no ground-truth illuminant color dataset exists for scenes containing multiple illuminants. We evaluated the proposed illuminant estimation method on two widely used publicly available ground-truth databases. The error between ground truth $\Gamma_{l}$ and estimated illuminant color $\Gamma_{e}$ is typically measured as angular error in RGB-space, defined as

$$
e=\cos ^{-1}\left(\frac{\Gamma_{l} \cdot \Gamma_{e}}{\left\|\Gamma_{l}\right\|\left\|\Gamma_{e}\right\|}\right)
$$

Somewhat unusual compared to other fields of computer vision, the success of illuminant-estimation methods is typically measured using the median over multiple images [16].
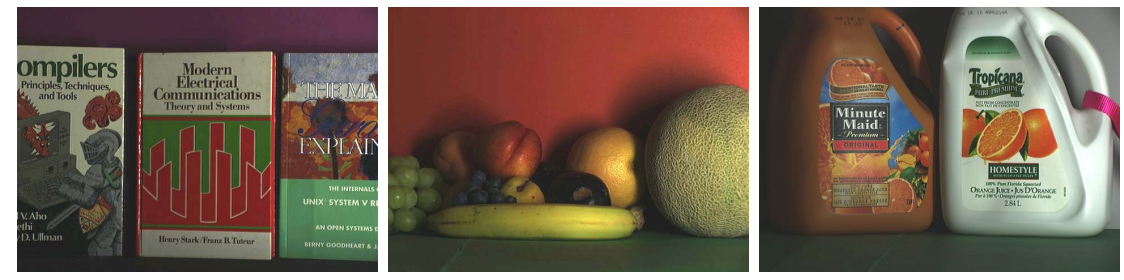

Fig. 4. Examples of benchmark laboratory images by [1].

Table 1. Algorithm performance on benchmark laboratory images (left) and on realworld images (right). The results are taken from [14,28].

\begin{tabular}{|l|c|}
\hline Method & Median $e$ \\
\hline \hline Gamut mapping & $\mathbf{3 . 1}^{\circ}$ \\
Gray-World & $8.8^{\circ}$ \\
White-Patch & $5.0^{\circ}$ \\
Color-by-Correlation & $8.6^{\circ}$ \\
\hline Proposed method & $4.4^{\circ}$ \\
\hline
\end{tabular}

\begin{tabular}{|l|c|}
\hline Method & Median $e$ \\
\hline \hline Regular gamut with offset-model & $5.7^{\circ}$ \\
Gray-World & $7.0^{\circ}$ \\
White-Patch & $6.7^{\circ}$ \\
Color-by-Correlation & $6.5^{\circ}$ \\
$1^{\text {st }}$-order Gray-Edge & $5.2^{\circ}(*)$ \\
$2^{\text {nd } \text {-order Gray-Edge }}$ & $5.4^{\circ}(*)$ \\
Tan et al. [39] & $5.6^{\circ}$ \\
\hline Proposed method & $\mathbf{4 . 4}^{\circ}$ \\
\hline
\end{tabular}

The first dataset, introduced by Barnard et al. [1], contains high-quality laboratory images. We used the "dielectric specularities" part of the dataset. It 

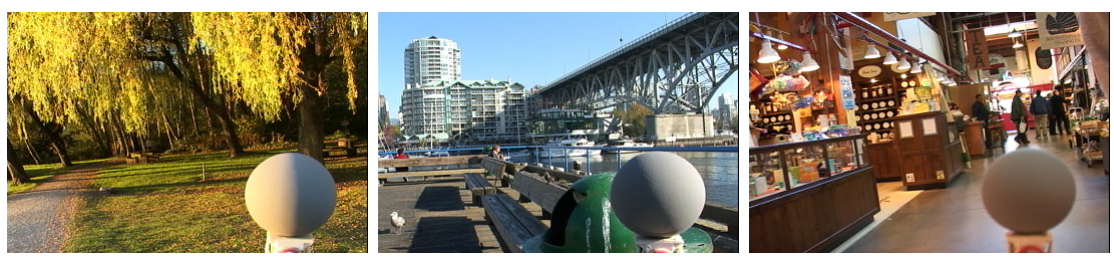

Fig. 5. Examples of benchmark real-world images.

contains 9 scenes, each under 11 different illuminants, since this part contains a mixture of specular and diffuse pixels. Example images are shown in Fig. 4. The proposed method performs comparable to other state-of-the art illuminant estimation methods, as shown in Table 1 (left). The results from the other methods were taken from [34].

The second dataset, presented by Ciurea and Funt [5], contains a wide variety of real-world scenes. The ground truth is estimated from a fixed matte gray ball that is mounted in front of the camera, as shown in Fig. 5. For the evaluation of the methods, the ball has to be masked out. Table 1 (right) shows that the proposed method is highly competitive on this dataset. The results marked with $\left.{ }^{*}\right)$ are taken from [28], the remaining results are from [14].

\subsection{Exposing Digital Forgeries}

For qualitative results on multiple illuminants, we collected from various sources, mostly flickr [11], approximately 430 images containing scenes with multiple illuminants or unusual single-illuminant setups. Besides these images, which were assumed (or known, respectively) to be original, 10 forgeries have also been examined using the proposed method. In the following, we present three cases where image geometry and illumination create discontinuities. Fig. 6 shows a case where the change in the illumination color is barely explicable with the scene setup. Both the illumination map as well as the distance map exhibit a sharp transition between the two persons in the foreground and the third in the back, which could only be feasible if there was a greater distance between them.

The example in Fig. 7 shows outdoor illumination with one dominant illuminant. Again, we compare the skin regions of the people, in order to have roughly comparable object materials. The selected regions are the directly lit skin of the inserted person versus the directly lit skin of other guests. The illumination map shows blueish estimates for the inserted man. The distance map makes this difference even more visible. Note that the estimates of the coast line in the background should be ignored (although they fit well, in this particular case). The underlying pixels must be assumed to be purely diffuse, and thus do not satisfy our underlying assumptions.

Fig. 8 contains a more complex case. The woman in the right is inserted in the image. Illumination map and distance map are plausible, compared to 

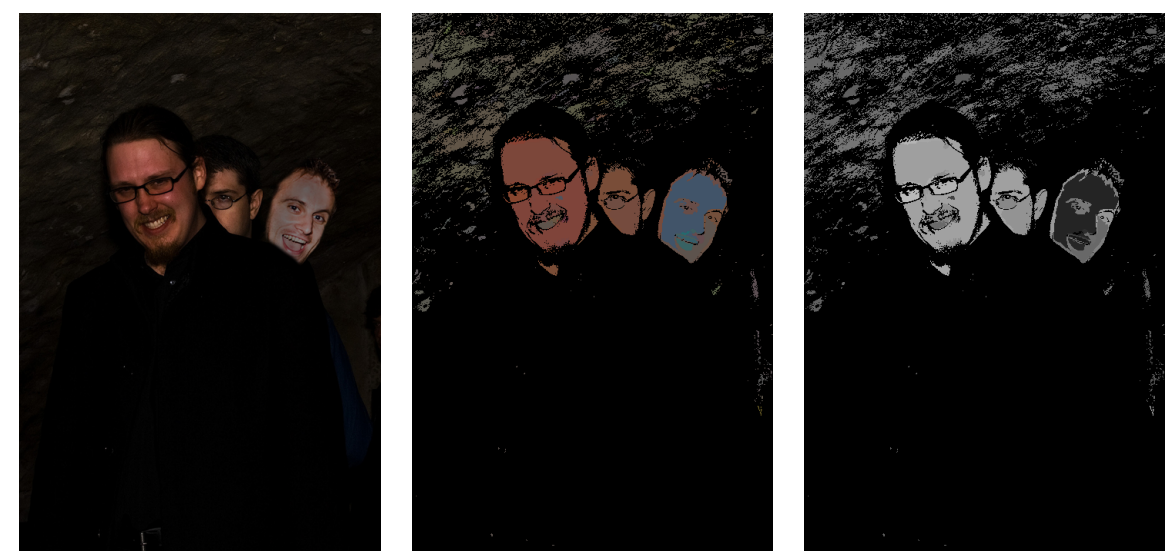

Fig. 6. Tampered image. Illumination map as well as distance map show a clear difference between the first two and the third person. Since the three stand close together in the image, it can be assumed that this difference is due to tampering.

the people that stand similarly close to the restaurant. However, adding again the scene geometry gives a strong clue that this scene is not original. Since the woman is turned away from the restaurant, the illuminant color on the woman's chest should share greater similarity with the body parts of the other people that are turned away from the restaurant lights.

\section{Conclusion}

We presented a method that estimates the color of the illuminant locally and applied it to the detection of tampered images. A user interactively marks regions whose illuminants should be further investigated. On these regions, the illuminant color is estimated. Then, the local illuminant estimation process is extended to the whole image. In scenes with multiple illuminants, one can typically observe a transition between these illuminants that is consistent with the scene geometry. In order to verify this, we introduced the illuminant map, consisting of all local illuminant estimates, and a distance map, that captures the influence of every illuminant. If an image has been manipulated, the transition between these illuminants should accordingly be disturbed.

This is preliminary work. In the future, we will extend this approach in two directions. First, the user interaction can further be reduced by postprocessing of the illuminant map and the distance map. Secondly, we aim to develop more rigorous methods for a more detailed analysis of inconsistencies in the illumination map and the distance map. 

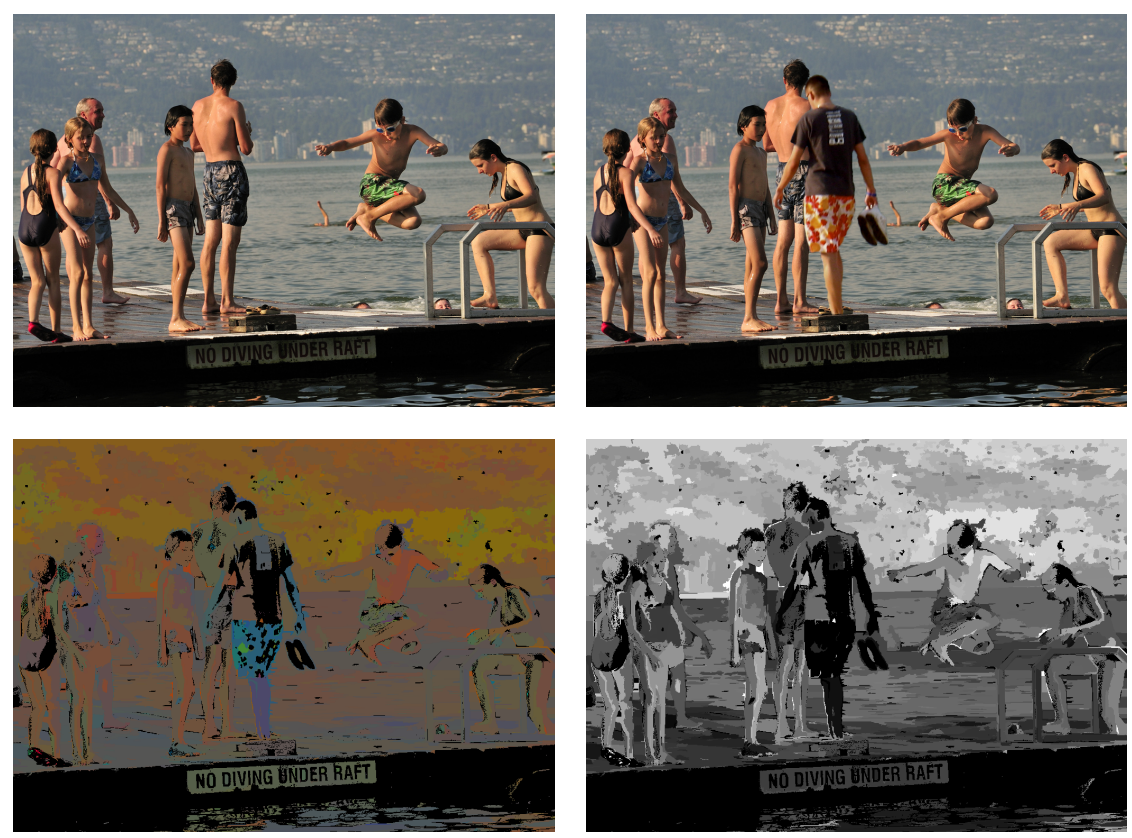

Fig. 7. Original image (top left) and tampered image (top right). A comparison of the skin regions of the people exposes the inserted man in the distance map.

\section{References}

1. Barnard, K., Martin, L., Funt, B., Coath, A.: A Data Set for Color Research. Color Research and Application 27(3), 147-151 (2002)

2. Bayram, S., Sencar, H., Memon, N.: An efficient and robust method for detecting copy-move forgery. In: Acoustics, Speech, and Signal Processing. pp. 1053-1056 (2009)

3. Bravo-Solorio, S., Nandi, A.: Passive Forensic Method for Detecting Duplicated Regions Affected by Reflection, Rotation and Scaling. European Signal Processing Conference (2009)

4. Cardei, V.C., Funt, B., Barnard, K.: Estimating the Scene Illumination Chromaticity Using a Neural network. Journal of the Optical Society of America A 19(12), 2374-2386 (2002)

5. Ciurea, F., Funt, B.: A Large Image Database for Color Constancy Research. In: Color Imaging Conference. pp. 160-164 (2003)

6. Dirik, A.E., Bayram, S., Sencar, H.T., Memon, N.: New features to identify computer generated images. In: IEEE International Conference on Image Processing. pp. 433-436 (2007)

7. Farid, H.: Exposing Digital Forgeries from JPEG Ghosts. IEEE Transactions on Information Forensics and Security 1(4), 154-160 (2009)

8. Felzenszwalb, P.F., Huttenlocher, D.P.: Efficient Graph-based Image Segmentation. International Journal of Computer Vision 59(2), 167-181 (2004) 

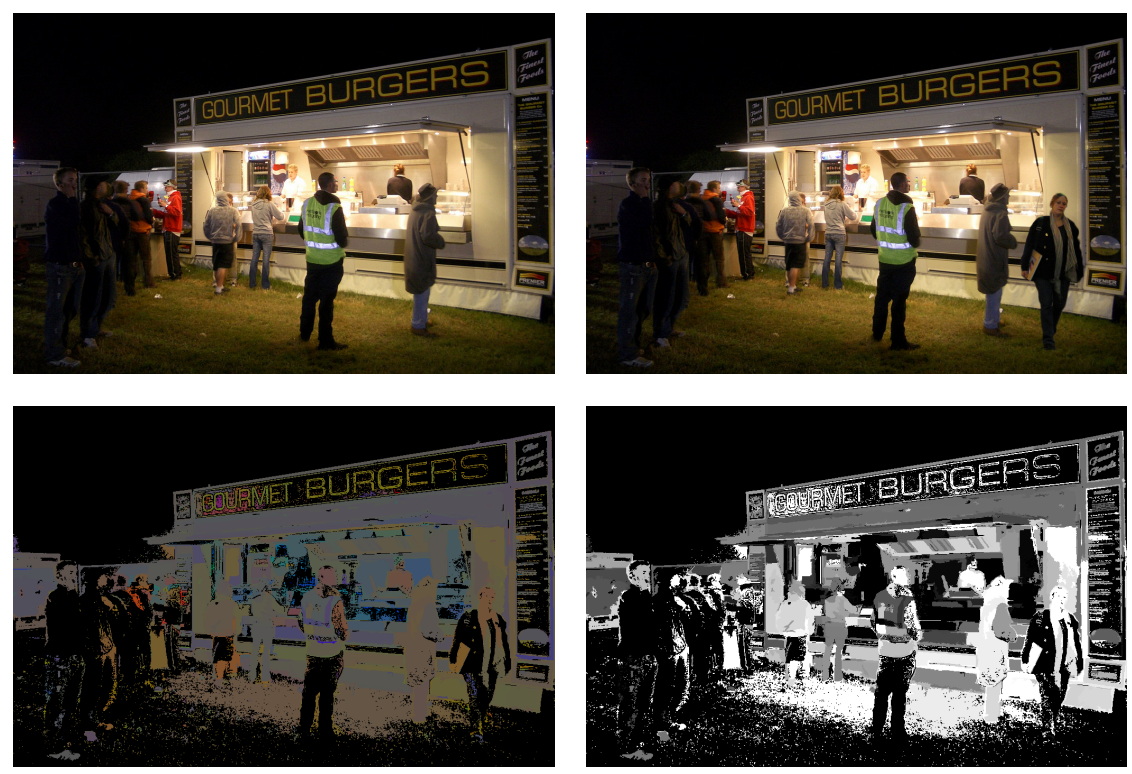

Fig. 8. Original image (top left) and tampered image (top right). At first glance, illumination map and distance map show plausible results on the tampered image. However, the illuminant estimates are obtained from the front of the woman, which is turned away from the restaurant lights. Therefore, the expected illumination should be more bluish, like e.g. at the back of the person in the middle.

9. Finlayson, G.D., Hordley, S.D., Hubel, P.M.: Color by Correlation: A Simple, Unifying Framework for Color Constancy. IEEE Transactions on Pattern Analysis and Machine Intelligence 23(11), 1209-1221 (2001)

10. Finlayson, G.D., Hordley, S.D., Tastl, I.: Gamut Constrained Illuminant Estimation. International Journal of Computer Vision 67(1), 93-109 (2006)

11. Flickr: http://www.flickr.com

12. Gallagher, A., Chen, T.: Image Authentication by Detecting Traces of Demosaicing. In: Computer Vision and Pattern Recognition Workshops. pp. 1-8 (2008)

13. Geusebroek, J.M., Boomgaard, R., Smeulders, A., Gevers, T.: Color Constancy from Physical Principles. Pattern Recognition Letters 24(11), 1653-1662 (2003)

14. Gijsenij, A., Gevers, T., van de Weijer, J.: Generalized Gamut Mapping using Image Derivative Structures for Color Constancy. International Journal of Computer Vision 86(2-3), 127-139 (2010)

15. He, J., Lin, Z., Wang, L., Tang, X.: Detecting Doctored JPEG Images Via DCT Coefficient Analysis. In: European Conference on Computer Vision. vol. 3, pp. 423-435 (2006)

16. Hordley, S.D., Finlayson, G.D.: Re-evaluating Color Constancy Algorithm Performance. Journal of the Optical Society of America A 23(5), 1008-1020 (2006)

17. Hsu, Y., Chang, S.: Image Splicing Detection using Camera Response Function Consistency and Automatic Segmentation. In: International Conference on Multimedia and Expo. pp. 28-31 (2007) 
18. Johnson, M., Farid, H.: Exposing Digital Forgeries through Specular Highlights on the Eye. In: International Workshop on Information Hiding. pp. 311-325 (2007)

19. Johnson, M.K., Farid, H.: Exposing Digital Forgeries by Detecting Inconsistencies in Lighting. In: Workshop on Multimedia and Security. pp. 1-10 (2005)

20. Johnson, M.K., Farid, H.: Exposing Digital Forgeries through Chromatic Aberration. In: Multimedia and Security. pp. 48-55 (2006)

21. Johnson, M.K., Farid, H.: Exposing Digital Forgeries through Chromatic Aberration. In: ACM Workshop on Multimedia and Security. pp. 48-55 (2006)

22. Kharrazi, M., Sencar, H.T., Memon, N.: Blind Source Camera Identification. In: IEEE International Conference on Image Processing. pp. 709-712 (2004)

23. Kirchner, T., Böhme, R.: Hiding Traces of Resampling in Digital Images. Information Forensics and Security 3(4), 582592 (Dec 2008)

24. Klinker, G.J., Shafer, S.A., Kanade, T.: The Measurement of Highlights in Color Images. International Journal of Computer Vision 2(1), 7-26 (1992)

25. Lalonde, J.F., Efros, A.A.: Using Color Compatibility for Assessing Image Realism. In: IEEE International Conference on Computer Vision (2007)

26. Lee, H.C.: Method for Computing the Scene-Illuminant Chromaticity from Specular Highlights. Journal of the Optical Society of America A 3(10), 1694-1699 (1986)

27. Lin, S., Gu, J., Yamazaki, S., Shum, H.Y.: Radiometric Calibration from a Single Image. In: IEEE Conference on Computer Vision and Pattern Recognition. pp. 938-945 (2004)

28. Lu, R., Gijsenij, A., Gevers, T., Nedovic, V., Xu, D., Geusebroek, J.M.: Color Constancy using 3D Scene Geometry. In: IEEE International Conference on Computer Vision (2009)

29. Lukáš, J., Fridrich, J.: Estimation of Primary Quantization Matrix in Double Compressed JPEG Images. In: Digital Forensics Research Workshop (2003)

30. Lukáš, J., Fridrich, J., Goljan, M.: Digital Camera Identification From Sensor Pattern Noise. Information Forensics and Security 1(2), 205-214 (2006)

31. Luo, W., Huang, J., Qiu, G.: Robust Detection of Region-Duplication Forgery in Digital Images. In: Pattern Recognition. vol. 4, pp. 746-749 (2006)

32. Mahdian, B., Saic, S.: Detection of Copy-Move Forgery using a Method Based on Blur Moment Invariants. Forensic Science International 171(2), 180-189 (2007)

33. Ng, T., Chang, S., Lin, C., Sun, Q.: Passive-Blind Image Forensics. In: Multimedia Security Technologies for Digital Rights, chap. 15, pp. 383-412. Academic Press (2006)

34. Personal communication: Arjan Gijsenij, University of Amsterdam

35. Popescu, A., Farid, H.: Exposing Digital Forgeries by Detecting Traces of Resampling. Signal Processing 53(2), 758-767 (Feb 2005)

36. Riess, C., Angelopoulou, E.: Physics-Based Illuminant Color Estimation as an Image Semantics Clue. In: International Conference on Image Processing (2009)

37. Sencar, H., Memon, N.: Overview of State-of-the-art in Digital Image Forensics. Algorithms, Architectures and Information Systems Security pp. 325-344 (2008)

38. Shafer, S.A.: Using Color to Separate Reflection Components. Journal Color Research and Application 10(4), 210-218 (1985)

39. Tan, R., Nishino, K., Ikeuchi, K.: Color Constancy through Inverse-Intensity Chromaticity Space. Journal of the Optical Society of America A 21(3), 321-334 (2004)

40. Yu, H., Ng, T.T., Sun, Q.: Recaptured Photo Detection Using Specularity Distribution. In: IEEE International Conference on Image Processing. pp. 3140-3143 (2008) 